\title{
We 0106
}

Multi Stage Fracking Optimization in Naturally

Fractured Reservoirs - A Discrete Numerical

Approach

J. Alvarellos* (Repsol), I. Aliguer (Universitat Politècnica de Catalunya), D. Garolera (Universitat Politècnica de Catalunya), I. Carol (Universitat Politècnica de Catalunya), M.R. Lakshmikantha (Repsol) \& J.M. Segura (Repsol) 


\section{Introduction}

In the case of low permeability reservoirs, hydraulic fracturing is a completion strategy that increases the contact between the well and the reservoir that finally will increase the well production. Fracture propagation is a result of the interaction of different physical processes like creation of new surface (fracture toughness), fluid flow in the fracture and porous media and their interaction, fracture geometry, rock elasto-plastic behavior, pre-existing fracture sets and in situ stress. The creation of a new surface enhances the permeability of the system but at the same time changes the in situ stress in the near field (close to the fracture). Those stress variations can affect fracture propagation itself (creation of conjugate fractures) or the fracture geometry in the case of multi fracture jobs. The existence of a naturally fractured system also can affect the fracture propagation. The fracturing fluid can move into the fractured media without creation of new surface just using, reopening and/or shearing the existing fracture sets. The interaction between the flow and the mechanical behaviors of hydraulic fractures propagating in a naturally fractured medium requires advanced numerical techniques that are able to reproduce all the interplaying aspects.

A particular application of the finite element method that explicitly represents the fractures by means of interface elements is used to explore the behavior of two cases: the fracture propagation in naturally fractured media and the interaction between different hydraulic fractures in a multi-fracture well stimulation. The used method considers a full coupling between flow of the fracking fluid in the fracture and in the porous media and the nonlinear stain-stress behavior of the fracture and the rock material. The numerical formulation allows the representation of pre-existing and developing fractures using zero-thickness interface elements and incorporating the appropriate constitutive model in each case: rock mechanics based for pre-existing fractures and fractured mechanics based for fractures in propagation. For the simulation of a hydraulic fracture, a disadvantage of the method could be that the potential fracture path needs to be predefined, although this can be overcome by using special remeshing techniques or by inserting interface elements along all the mesh elements faces.

The first case studies the interaction between hydraulic fractures. A theoretical comparison between different fracturing sequences is presented. In the second case a hypothetical simple study of a naturally fractured media is presented. In this application example it is clear that not only the minimum horizontal stress controls the fracture propagation but also, the topology of the natural fracture system and the shear stresses acting on it are important parameters to define fracture growth. 